\title{
Coexistence of a Congenital Arteriovenous Fistula of the Left Breast with a True Aneurysm of the Right Internal Mammary Artery
}

\author{
Ahmet Said Çil; Murat Bozkurt; Duygu Kara Bozkurt; and Adem Karabacak
}

Arteriovenous fistulas (AVF) and true aneurysms are uncommon arterial vascular disorders of the breast. The etiology can be either acquired or congenital. Coexistence of a congenital AVF and true aneurysm of internal mammary artery (IMA) branches is a very rare condition. We present a case of congenital AVF and true aneurysm of the IMA in a woman, age 56 years. To the best of our knowledge, this is the first published case of the coexistence of a congenital AVF with a true aneurysm of the breast. The radiologic findings of these rare entities have been reviewed according to the literature.

Keywords: Aneurysm; Arteriovenous fistula; Breast; Doppler ultrasonography; Magnetic resonance imaging

Corresponding Author: Murat Bozkurt, MD

Universal Malatya Hastanesi

Turgut Özal Bulvarı

Ankara Asfaltı 6 km No:219

44000 Malatya / Turkey

Tel: $904222382828 / 1546$

Tel2: 905322279072

Fax: 904222382600

Email: jindrmb@yahoo.com

Received: January 16, 2013

Revised: April 4, 2013

Accepted: April 10, 2013

doi: $10.3121 / \mathrm{cmr} .2013 .1147$ ascular abnormalities of the breast include a wide spectrum of arterial and venous disorders as well as benign and malignant vascular masses. Multimodality imaging is often necessary to accurately diagnose several of these uncommon conditions, and pathologic confirmation can be important for accurate diagnosis of vascular masses. The arterial supply to the breast is primarily derived from branches of the internal mammary (thoracic) artery (IMA), intercostal arteries, and the lateral thoracic artery. The IMA is the dominant artery supplying the breast, and its mammarian branches supply the medial and central breast parenchyma. The lateral thoracic artery supplies the superolateral breast parenchyma. ${ }^{1}$ Arterial vascular diseases of the breast include atherosclerosis, true and pseudo-aneurysm, congenital and acquired arteriovenous fistula (AVF), and benign and malignant vascular neoplasias (hemangioma, angiolipoma, angiosarcoma, hemangiopericytoma, etc.). Atherosclerotic vascular disease of the breast is most commonly seen on a daily basis in screening mammography. Coexistence of congenital AVF and true aneurysm of the IMA is a very rare condition.

Multiple radiologic modalities are often used to pre-diagnose several of these uncommon conditions, and pathologic confirmation can be important for the accurate diagnosis of vascular masses. Radiologic modalities include mammography, color Doppler sonography, and dynamic contrast-enhanced magnetic resonance imaging (MRI).

A case of congenital AVF of the left internal thoracic artery and true aneurysm of the right internal thoracic artery are described in a woman, age 56 years. An AVF 

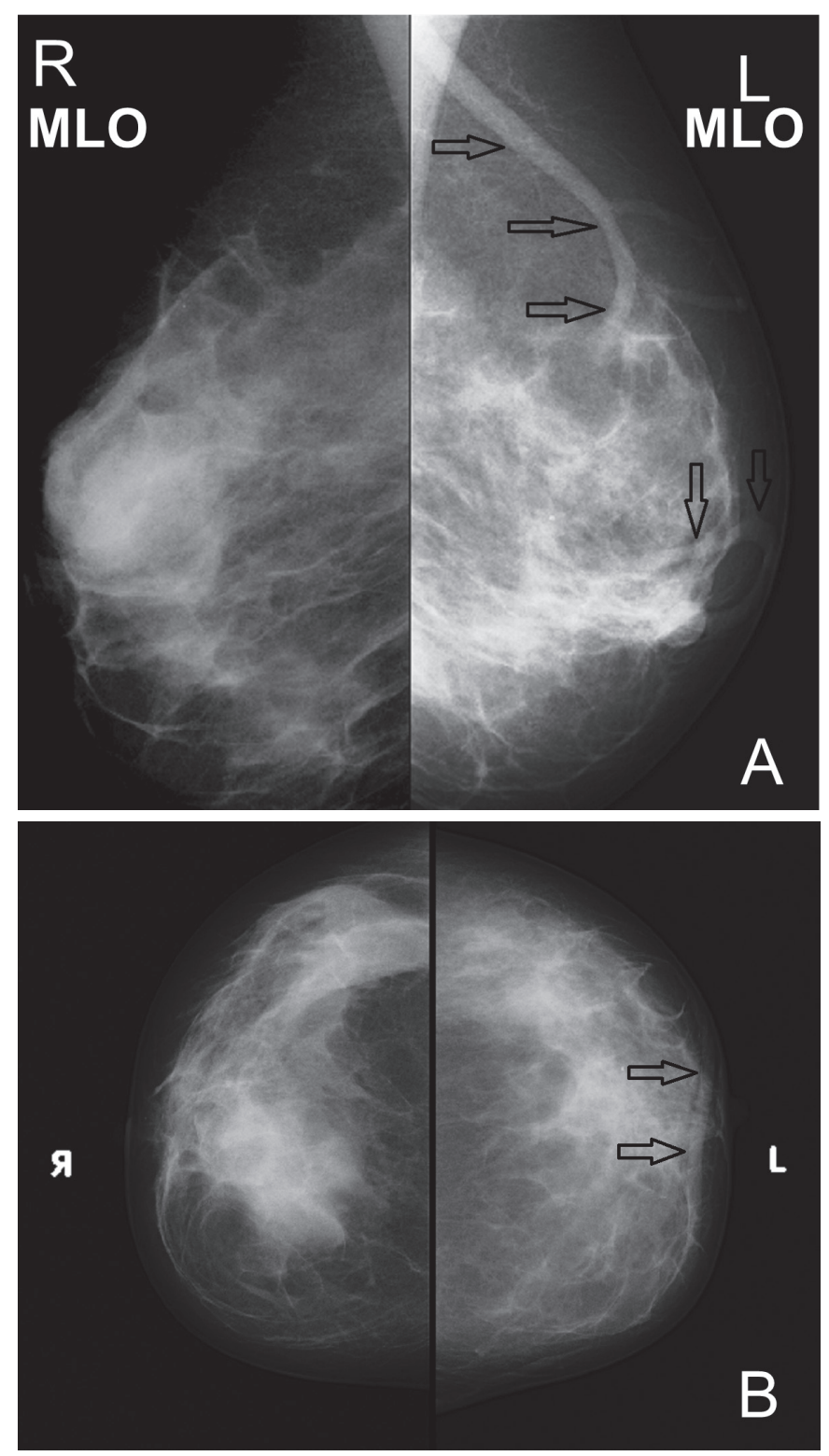

Figure 1: (A) Mediolateral oblique (MLO) and (B) craniocaudal mammography views of both breasts demonstrating dilated vascular structure in the retroareolar region and axillary localization of the left breast (open arrows).

was seen in the left breast with communication occurring between the second medial mammary branch of the left IMA and a superficial venous structure of left breast with a true aneurysm occurring from the first anterior perforating branch of the right IMA. Clinical features, mammographic, sonographic evaluation, and MRI findings of this rare entity are presented through a review of the literature.

\section{Case Report}

A woman, age 56 years, gravida 3, para 3, was admitted to the Gynecology and Obstetrics Clinic of the Universal Malatya Hospital with nonspecific, intermittent, bilateral breast pain for the past two years. It was more pronounced in the upper quadrants when the patient was lying down, especially in the prone position.
The patient had no history of chest trauma or surgery and did not use hormone replacement therapy. There was no history of chronic disease such as hypertension, diabetes mellitus, or heart failure. On physical breast examination, dilated venous structures were seen on the upper quadrants of the left breast, and a systolic thrill was palpated on the upper medial quadrant of the left breast. Vaginal examination revealed a normal vulva and vagina. There was cervical erosion measuring $2 \mathrm{~cm} \times 2 \mathrm{~cm}$, with endometrial thickness of $4 \mathrm{~mm}$ in the middle of the endometrium. A Pap smear was performed and was normal.

The patient had no risk factors for breast cancer (no positive family history, BRCA gene mutation, previous chest radiation, hormone therapy, or tobacco smoking). Because of her symptoms, age, and positive physical breast examination, a mammography was performed (figure 1) and demonstrated
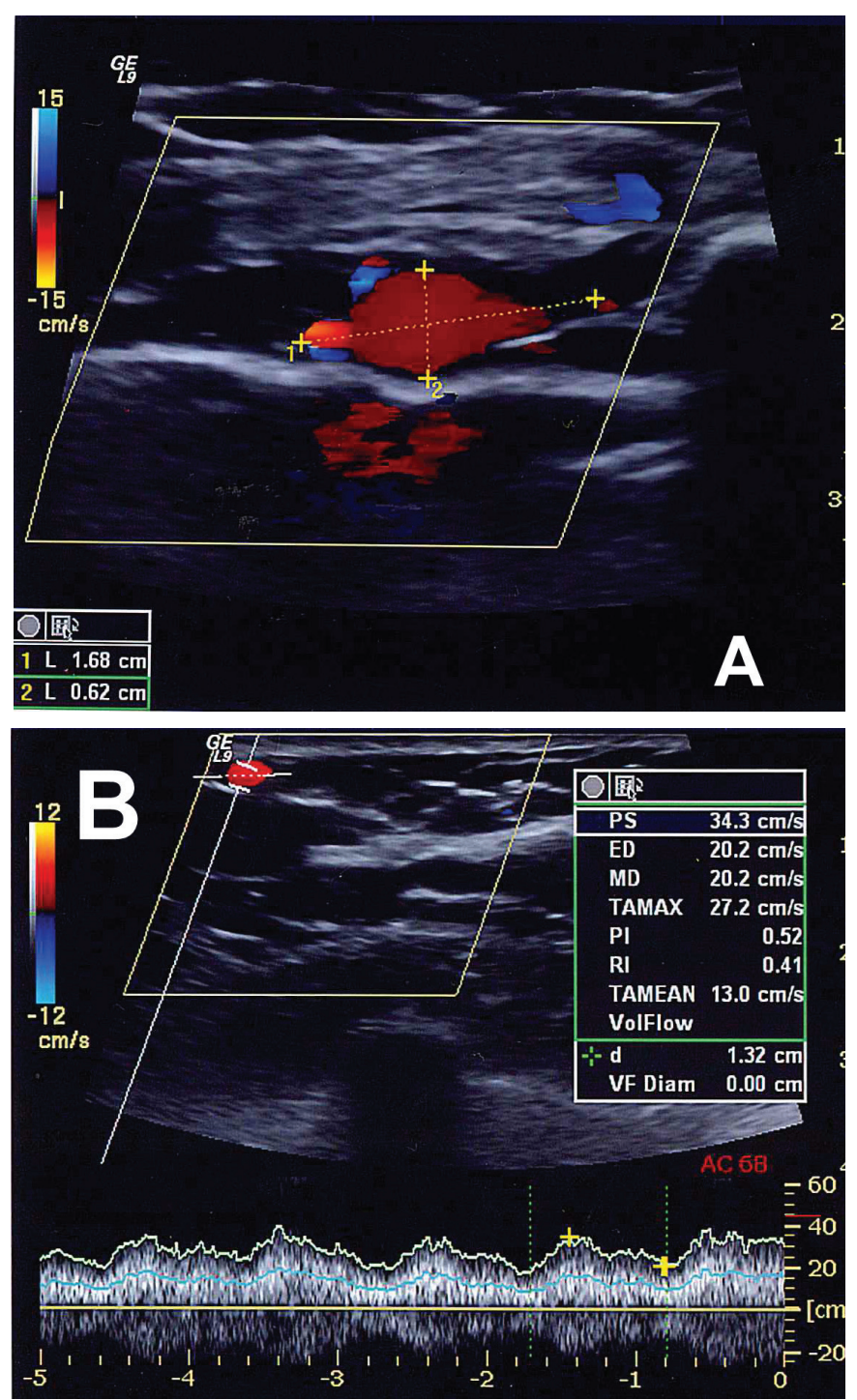

Figure 2: Color Doppler ultrasound images demonstrating a fusiform shaped $16 \mathrm{~mm} \times 6 \mathrm{~mm}$ diameter aneurysm in the right breast $(A)$ and an arterialized venous wave form (RI 0.41) inside the proximal segment of the draining vein of the arteriovenous fistula in the left breast (B). 

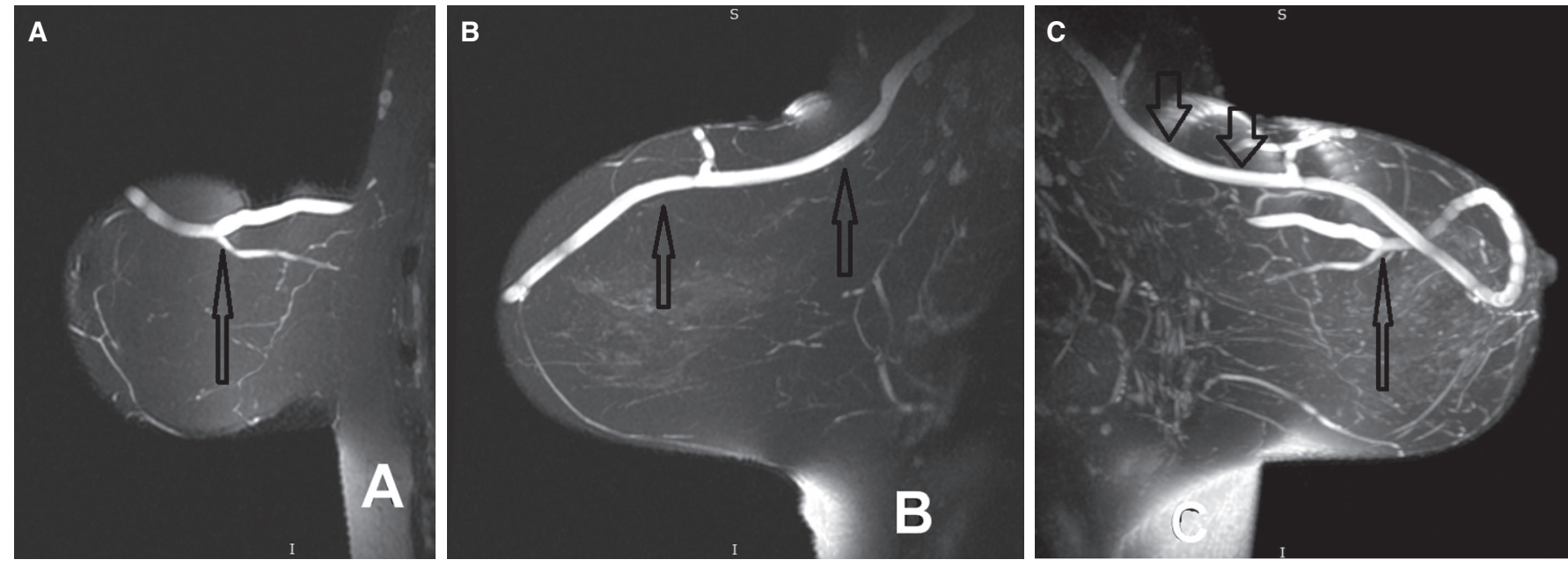

Figure 3: (A, B) Sagittal fat-suppressed T2-weighted images and (C) maximum intensity projection images that were obtained from sagittal T2-weighted images of the left breast showing the connection of arterial and venous structure and dilated draining vein extending into the axillary region (open arrows).

dilated venous structures in the retroareolar and axillary region of the left breast.

An ultrasound of the breast was performed with a LOGIQ 9 ultrasound system (GE Healthcare Technologies, Ultrasound, Milwaukee, WI, USA) by using a linear (12-14 MHz) transducer. On ultrasound, a $7 \mathrm{~mm}$ diameter, dilated, superficial venous structure located in the subcutaneous tissue was seen in the upper medial quadrant and crossing to the lateral quadrant of the left breast, draining toward the axillary vein. On color Doppler examination, AVF was diagnosed when arterializated venous waveform (RI 0.41 and PI 0.52) was seen, and communication between an artery and a vein was demonstrated in the upper medial quadrant of the left breast. In the upper medial quadrant of the right breast, a fusiform shaped aneurysm, measuring $16 \mathrm{~mm}$ x $6 \mathrm{~mm}$, was observed (figure 2). Based on the Doppler sonographic findings of the breast, an MRI examination was planned to confirm the diagnosis of AVF and the aneurysm.

Dynamic contrast enhanced breast MRI was performed on a 1.5-Tesla clinical MR system (General Electric Signa Excita, GE Health care system, Milwaukee, WI, USA). The patient was placed in the prone position, and a dedicated phasedarray bilateral four-channel breast coil (General Electric MRI devices, WI, USA) was used for imaging the breasts. The MRI scan protocol included axial T1-weighted fast spin echo (TR/TE, 560/7 msec), axial T1-weighted three-dimensional fast-spoiled gradient-echo fat-suppressed sequence (TR/TE 3.3/1 msec; flip angle $13^{\circ}$; bandwidth, 62,5), and axial and sagittal T2-weighted fat-suppressed fast spin echo series (TR/ TE, 5000/85 msec). Finally, dynamic contrast-enhanced fatsuppressed T1-weighted gradient echo images (TE/TR 3.3/1 msec; flip angle 13, dynamic scan duration $60 \mathrm{sec}$ ) were acquired before and immediately after administration of 0.1 $\mathrm{mmol} / \mathrm{kg}$ gadolinium-based contrast agent to study the contrast enhancement of the lesions.
The MRI showed dilated venous structures in the upper quadrant of the left breast and dilated fusiform shaped arterial structure in the upper medial quadrant of the right breast. Maximum intensity projection (MIP) MRI illustrated an AVF in the left breast with communication occurring between the second medial mammary branch of the left IMA and left superficial mammarian venous structure with drainage toward the axillary vein (figure 3). In the upper medial quadrant of the right breast, a fusiform aneurysm was seen that measured $16 \mathrm{~mm} \times 10 \mathrm{~mm}$ in diameter, originating from the anterior perforating branch of the right IMA (figure 4).

We attempted to refer the patient to another hospital for catheter angiography, because a catheter angiography unit is not yet established in our hospital. However, the patient did not agree to go to another hospital for angiography. The AVF was corrected surgically under general anesthesia, and the aneurysm was managed conservatively by thrombin injection.

\section{Discussion}

Vascular pathologies of the breast represent uncommon conditions, except for atherosclerotic disease of the breast. Vascular pathologies of the breast are classified into three categories: arterial, venous and mixed types AVFs, and true aneurysms of the breast, which are uncommon pathologies. The coexistence of a congenital AVF and true aneurysm of the breast is very rare.

An AVF is an abnormal communication between an artery and a vein. An AVF may be congenital (usually affecting smaller vessels) or acquired as a result of trauma or erosion of an arterial aneurysm into an adjacent vein. Aneurysms are divided into two categories - true or pseudo- aneurysms. True aneurysms of the breast are thought to be due to prior trauma. Pseudo-aneurysms are most often associated with trauma and are recognized as a complication of diagnostic interventional techniques. ${ }^{2}$ 

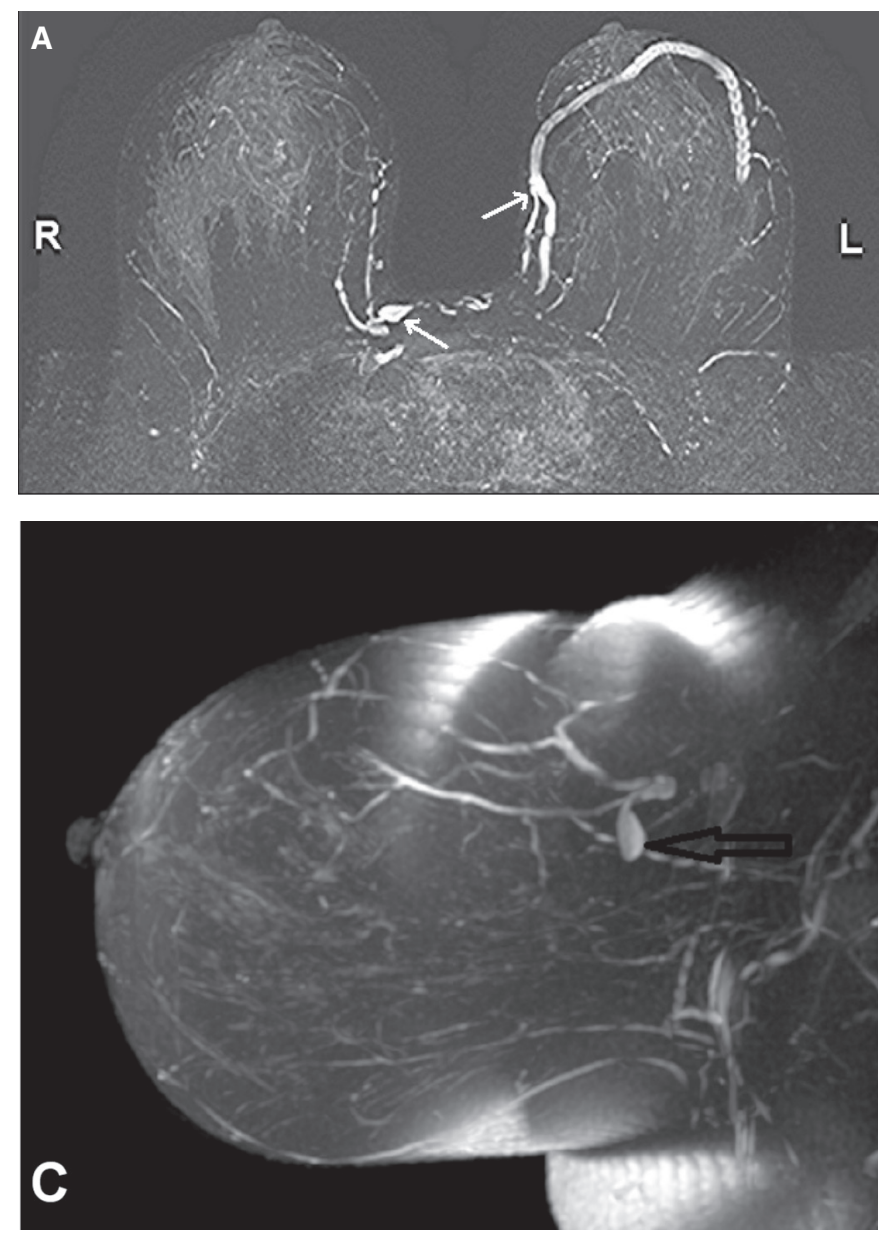

Figure 4: $(A)$ Maximum intensity projection images that were obtained using contrast-enhanced, axial T1-weighted fatsuppressed fast spoiled gradient-echo sequence showing an arteriovenous fistula in the left breast and an aneurysm near the right breast (arrows). (B) Sagittal fat-suppressed T2-weighted images. (C) Sagittal fat-suppressed T2-weighted maximum intensity projection image of the left breast showing an aneurysm (open arrows) on the first internal thoracic artery branch. (D) Axial short-tau inversion recovery image showing a $16 \mathrm{~mm} \times 7 \mathrm{~mm}$ diameter fusiform dilated vascular structure near the right breast.

In this case report, we describe a woman with a nontraumatic, presumably congenital AFV and true aneurysm of the breast originating from the internal thoracic artery. These vascular pathologies should be considered in the differential diagnosis of mastodynia. In our case, the patient presented with nonspecific, intermittent, bilateral breast pain, especially in the upper quadrants.

On physical breast examination, dilated venous structures were seen and systolic thrill was palpated in the left breast when the patient was lying down, especially in the prone position. Vlahos et $\mathrm{al}^{3}$ presented a case of congenital AVF in a young woman; the chief complaint was a murmur when lying down, especially in the prone position, a palpated lump, and the presence of varicose-like veins in the breast, similar to our patient. Vascular disorders of the breast represent a
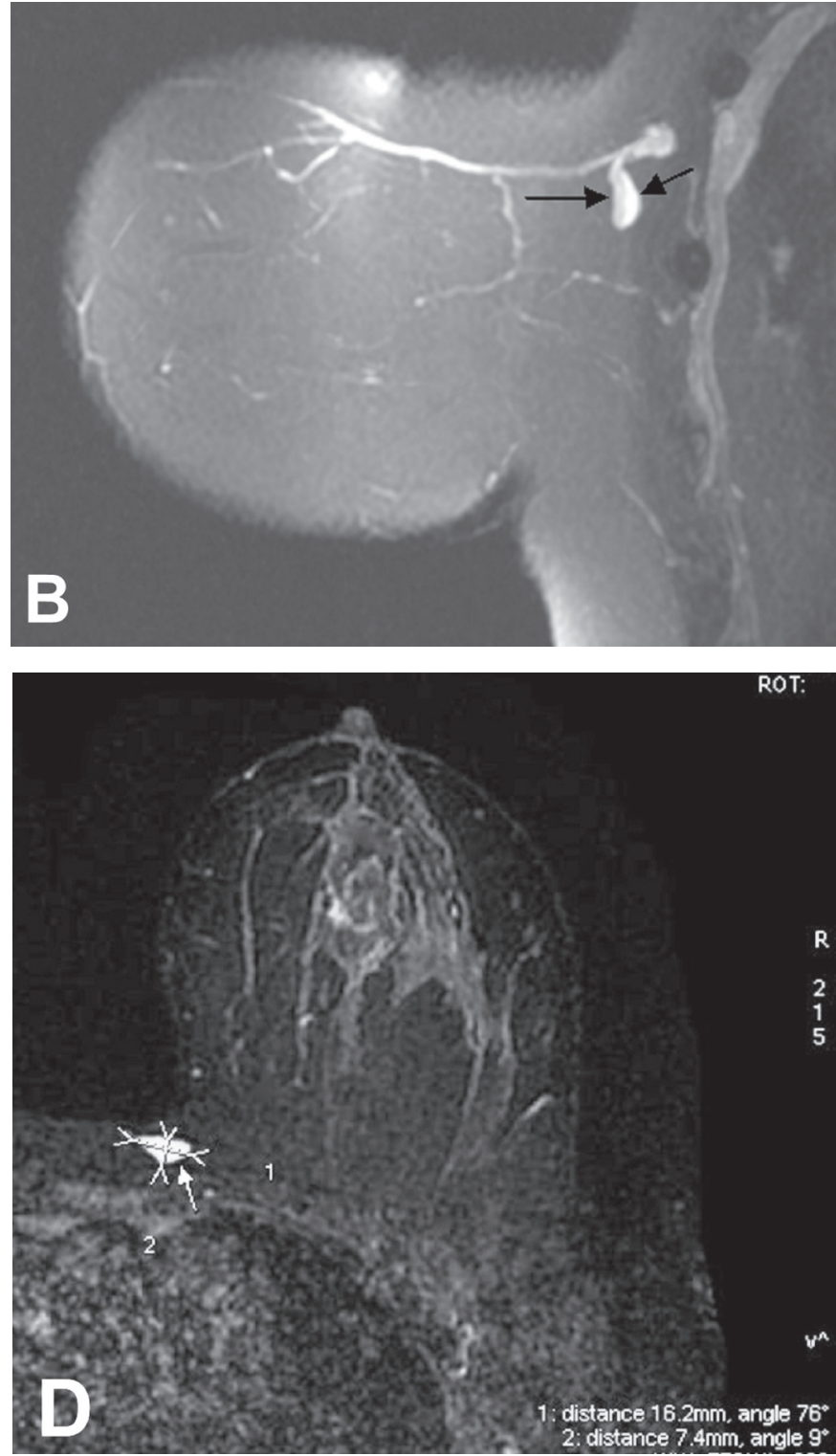

broad spectrum of conditions, and differential diagnosis is difficult using only one imaging modality. ${ }^{1}$ Multiple imaging modalities (eg, mammography, color Doppler ultrasound, and MRI) are used for the diagnosis of vascular breast pathologies. In our case, on color Doppler ultrasound, a fusiform shaped aneurysm in the right breast was demonstrated. In the left breast, an arterialized venous wave form inside the proximal segment of the draining vein of the AVF was observed. On MRI, connection of the arterial and venous structure of the AVF and a dilated draining vein that extended to the axillary region was demonstrated in the sagittal T2-weighted MIP images. In the right breast, the aneurysm appeared as a saccular form on MRI. The Doppler ultrasound confirmed the fusiform aneurysm that showed the arterialized venous wave form in the distal segment of the aneurysm. Localization of the pathologies and the connections of the AVF were illustrated very clearly using these imaging modalities for effective surgical treatment. To the best of our knowledge, this is the first case in the literature of the coexistence of congenital AVF and true aneurysm of the breast. 
Two congenital breast AVFs have been previously reported. ${ }^{3,4}$ Cox et $\mathrm{al}^{5}$ described multiple true aneurysms in the female breast in a patient with a history of chronic amphetamine abuse. They postulated that amphetamine abuse may have been a factor in the development of multiple breast aneurysms. The aneurysm was demonstrated on Doppler ultrasonography in this patient. ${ }^{5}$ Dehn and Lee $^{6}$ reported an AVF between the right second anterior intercostal artery and vein that occurred after an episode of hypertension crisis. The AVF was demonstrated on conventional angiography and treated by ligation of the artery and vein. Our patient did not agree to catheter angiography for diagnosis and treatment of her vascular pathologies. Pseudo-aneurysms of the breast occur relatively more frequently than the true aneurysms. There are several reports in the literature of pseudo-aneurysm and AVF formation in the female breast after invasive procedures, such as biopsy. ${ }^{7,8}$ Distinction between true and pseudo-aneurysm is made by histopathology. The surgically extracted pathological specimen from our case revealed true aneurysm of the breast vessels.

Dynamic contrast enhanced breast MRI with MIP images has become a standard imaging method for evaluation of breast disorders. ${ }^{9}$ Vessels are recurrently encountered in predictable locations, and venous collateral connections may be demonstrated on MIP images. Increased asymmetric vascularity of the breast has also been found to be a potentially important marker for breast cancer. ${ }^{9}$ Knowledge of the vascular anatomy of the breast is important for surgeons performing excisions and breast reconstructive surgery. ${ }^{1}$

In conclusion, breast MRI is superior to other conventional breast imaging methods for detection of vascular disorders of the breast. Color Doppler ultrasound may be required to confirm the diagnosis. A noninvasive imaging modality, such as MRI, can be used in the diagnosis of breast vascular disease, especially in patients who do not agree to catheter angiography.

\section{References}

1. Jesinger RA, Lattin GE Jr, Ballard EA, Zelasko SM, Glassman LM. Vascular abnormalities of the breast: arterial and venous disorders, vascular masses, and mimic lesions with radiologic-pathologic correlation. Radiographics 2011;31:E117-E136

2. Dixon AM, Enion DS. Pseudoaneurysm of the breast: case study and review of literature. Br J Radiol 2004;77:694-697.

3. Vlahos L, Prunzos P, Kailidou E, Gouliamos A, Papacharalamous X, Papavasiliou C. Congenital A-V fistula of the breast. Radiologe 1991;31:250-252.

4. Hurt RL, Wells BG. Congenital arteriovenous fistula of the internal mammary vessels. Br Heart J 1957;19:135-137.

5. Cox J, Kaye B, Burn D, Bliss R. Multiple aneurysms in the female breast: a case report. Br J Radiol 2007;80:e275-e277.

6. Dehn TC, Lee EC. Aneurysm presenting as a breast mass. Br Med J (Clin Red Ed) 1986;292:1240.

7. Smith SM. Breast pseudoanerysm after core biopsy. AJR Am J Roentgenol 1996;167:817.

8. Chorny K, Raza S, Bradley FM, Baum JK. Pseudoaneurysm formation in the breast after needle core biopsy. J Ultrasound Med 1997;16:849-851.
9. Kul S, Cansu A, Alhan E, Dinc H, Reis A, Çan G. Contrastenhanced MR angiography of the breast: Evaluation of ipsilateral increased vascularity and adjacent vessel sign in the characterization of breast lesions. AJR Am J Roentgenol 2010;195:1250-1254.

\section{Author Affiliations}

Ahmet Said Çil ; Murat Bozkurt ${ }^{*}$; Duygu Kara Bozkurt"; and Adem Karabacak

*Universal Hospitals Group, Department of Radiology, Malatya, Turkey

Universal Hospitals Group, Obstetrics and Gynecology

Department, Malatya, Turkey

${ }^{*}$ Sevgi Hospital, Department of Radiology, Malatya, Turkey

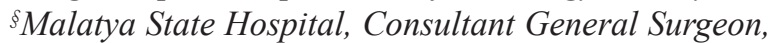
Malatya, Turkey 